\title{
Mitgestalter statt Feuerwehr
}

\author{
Schulsozialarbeit aus schulpädagogischer Sicht
}

Manfred Bönsch

Die Schule kann sich grundlegend nur ändern, wenn Lehrerinnen und Lehrer mit Sozialarbeiterinnen und Sozialarbeitern gleichberechtigt zusammen arbeiten. Nur wenn man die zu bearbeitenden Probleme gemeinsam identifiziert, wird man die Aufgabenfelder finden, die in einer Schule vordringlich $z u$ bearbeiten sind.

Schulsozialarbeiterinnen und Schulsozialarbeiter (Sozialpädagogen/Sozialarbeiter) sind heute an Hauptschulen fast flächendeckend, an Berufsschulen und Förderschulen in größerer Zahl, an Grundschulen bisher wenig, an Realschulen und Gymnasien kaum tätig. Häufig ist das Argument für ihren Einsatz schlicht darin zu sehen, dass Verhaltens- und Leistungsprobleme heute so stark auftreten, dass Lehrerinnen und Lehrer mit dieser Problemlage nicht fertig werden und der Nothelfer bedürfen.

Diese Nothelfer sind aber einer anderen Berufsgruppe zugehörig, sie haben andere Vorstellungen von Kinder- und Jugendarbeit und stehen der Schule mit ihrem Leistungsanspruch und ihrer Arbeit häufig skeptisch gegenüber. Lehrerinnen und Lehrer selbst haben ebenso häufig noch ein Verständnis von Schule, das eher dem Bild von der Unterrichtsanstalt folgt und Schülerinnen und Schüler schlicht ihrer Vorstellung von Leistungsansprüchen unterwirft. Die Lebensprobleme von Kindern und Jugendlichen werden eher als Störelement angesehen, statt sie als Ausgangspunkt möglichen oder eben nicht mehr möglichen schulischen Lernens anzusehen und sie als Herausforderung zu verstehen.

So besteht die Gefahr, dass Schulsozialarbeit als Feuerwehr, wenn es brennt, oder als Pannenhilfe angesichts dauern-

Prof. Dr. Manfred Bönsch leitete bis zu seiner Emeritierung an der Universität Hannover das Institut für Erziehungswissenschaft.

E-Mail boenschhannover@aol.com der Betriebsstörungen angesehen wird. Es kann sich schnell ein Dualismus einschleichen: Die Sozialpädagogen bearbeiten die (größten) Schwierigkeiten, damit die Lehrenden ungestörten Unterricht durchführen können. Institutionelle Probleme (das Verständnis von Schule) und ein eher alternatives Verständnis von Jugendhilfe, Selbstverständnisprobleme (hier Unterrichtende mit dem wichtigeren Auftrag, da jugendspezifische Ansätze und Methoden) sowie Besoldungsfragen können Diskrepanzen, Subordination, Hierarchie befördern. So sind konzeptionelle Fragen aktueller denn je, um Bruchstellen und überflüssige Komplikationen zu vermeiden.

\section{Das zentrale Anliegen: \\ Soziales Lernen und eine integrierte Antwort auf Lebens- und Lernprobleme}

Der archimedische Punkt, die Ausgangsfrage, für das konzeptionelle Bedenken von Schulsozialarbeit ist: In welcher Weise kann die Schule eine sozialpädagogische Schule sein und in welchem Ausmaß kann sie Verhaltens- und Leistungsinsuffizienzien nicht dauernd nur als Defizitphänomene betrachten, sondern positiv als Herausforderungen einer besseren pädagogischen Arbeit verstehen?

Positive Antworten führen $\mathrm{zu}$ einer Verschiebung der Wahrnehmungen. Nicht die Behinderung des alltäglichen Unterrichts ist der Punkt. Gesucht werden Antworten auf die Frage, warum Schülerinnen und Schüler Schwierigkeiten haben, sich auf die schulischen Anforderungen einzulassen. Die zentralen, zu bearbeitenden Probleme sind dann:

- Welche Lebensmilieus produzieren die Distanzen zu der als künstlich und lebensfremd empfundenen Schule?

- Warum funktionieren die Transmissionsriemen in Gestalt positiver Lernund Leistungseinstellungen (Werte- und Normenrahmen, gute Gewohnheiten, Regelorientierung, Leistungswille) vom Elternhaus und Lebensmilieu in die Schule hinein nicht?

- Welche fundamentalen sozialen Kompetenzen fehlen, um Lernarbeit positiv zu gestalten?

- Welche Einbrüche in den Bildungsbiografien (Sitzenbleiben, Schulwechsel nach unten, Schulbesuch ohne Abschluss) schaffen dysfunktionale Einstellungen zum Lernen?

- Welche Formen struktureller Gewalt, die die Schule selbst produziert, schaffen zunehmende Distanz zum Lernen (Anonymität, Monotonie, unbarmherzige Handlungsabläufe, ungerechte Leistungsbeurteilung u. a. m.)?

- Ist vielleicht das Lernen selbst nie gelernt worden, sodass man es eben auch nicht in die eigene Hand nehmen kann? Man kann es für sich selbst nicht organisieren.

- Sind Lernklima und die täglichen Interaktionsstandards eventuell so bedrükkend, dass man eigentlich nur flüchten kann (Schulverweigerung)?

Das heißt in der Summe: Was ein Individuum eigentlich trägt - zuverlässige Bindungen, helfende Menschen, befriedigender Alltag - und was Anregung und Aufmunterung in der Schule ausmachen müssten, sind nicht in genügendem Maß gegeben und die Ohnmachtsgefühle gegenüber der Schule nehmen überhand. Das Sich-Behaupten durch Verweigerung, die Abwendung von an sich positiven Herausforderungen (Lernen) führen dann zu Friktionen gegenüber den Erwartungen der Institution. Die Lebensund Lernlage ist überwiegend defizitär!

\section{Beziehungsarbeit und Sozialarbeit}

Klar ist, dass mit herkömmlichem Unterricht auf die skizzierten Probleme nicht angemessen reagiert werden kann. 
»Business as usual « ist nicht mehr möglich. Also erhebt sich die Frage, wie die Schule anders reagieren kann - wenn sie das will!

Der radikale Gedanke, Lehrerinnen und Lehrer nicht mehr nur zu Unterrichtenden auszubilden, sondern auch zu Pädagogen und Sozialpädagogen, ist gegenwärtig als utopisch anzusehen. Selbst wenn dies gewollt werden würde, wären die Konsequenzen in Bezug auf die Schule massiv. Man müsste beispielsweise daran denken, die wöchentliche Arbeitszeit auf die Vorbereitung, Durchführung und Analyse inhaltsorientierten Unterrichts und auf Zeiten sozialpädagogischer Tätigkeit (außerunterrichtliche Kultur- und Bildungsarbeit, Hilfen für benachteiligte und gefährdete Kinder und Jugendliche, Jugendberufshilfe) aufzuteilen. Die Zeitstrukturen der Schule wären zu ändern. In der Folge wären viel mehr Lehrende einzustellen. Eine sozialpädagogisch orientierte Schule nur mit der Berufsgruppe der Lehrerinnen und Lehrer verlangt erhebliche Ausbildungsanstrengungen und finanzielle Ressourcen. Man kann diesen Ansatz bedenken, aber kaum realisieren.

Dann kommt man zu der Frage zurück, inwieweit auf die Notwendigkeiten der Kooperation mit einer zweiten Berufsgruppe, den Sozialpädagogen und Sozialarbeitern, am besten reagiert wer- den kann. Pragmatisch-zynisch könnte man anmerken, dass dies ohnehin billiger ist! Auf dieses Problem der Asymmetrie in der Besoldung wird hier nicht eingegangen, obwohl es natürlich Status- und Hierarchiefragen massiv berührt.

Entscheidender ist, das konzeptionelle Denken zu befördern, um pure Ergän-
Was eine für Kinder und Jugendliche helfende Schule ausmacht, soll mit zwei Ideen-Landkarten deutlich gemacht werden (vgl. Grafiken). Daraus kann sich das konzeptionelle Bedenken speisen und zu einem entsprechenden Schulprogramm führen, das in Arbeitsteilung, Kooperation und in integrativer Absicht realisiert werden kann. Übersicht 1 führt die drei zen-

\section{»Nicht die Behinderung des alläglichen Unterrichts ist das Problem, sondern die Schwierigkeiten von Schülerinnen und Schüler, sich auf die schulischen Anforderungen einzulassen«}

zung im Sinne von Pannendienst oder Feuerwehr von der Schule her gesehen und Nischendenken oder Distanzmodelle auf der Seite der Jugendhilfe zu vermeiden. Die Gefahren des Nebeneinanders, der Subordination, gar der Gegenpädagogik - gegenüber den »harten « Leistungsfaktoren der Lehrerinnen und Lehrer kann man sich um die »weichen « Faktoren (netter Kontakt!) kümmern - sind zu vermeiden. tralen Bereiche auf: das Beziehungsangebot, die Hilfen zur Identitätsfindung und das Erwartungs- und Vertrauensklima. Beziehungsarbeit und Erziehungsarbeit haben als Kerninhalt die Hilfen zur Identitätsfindung in individueller und sozialer Perspektive. Der Weg zur Persönlichkeitsbildung, zu sich selbst ist lang, gelegentlich schwer, von Brüchen begleitet, er steht aber gegenüber allen profanen und hurtigen Leistungsanforderungen im Vorder-

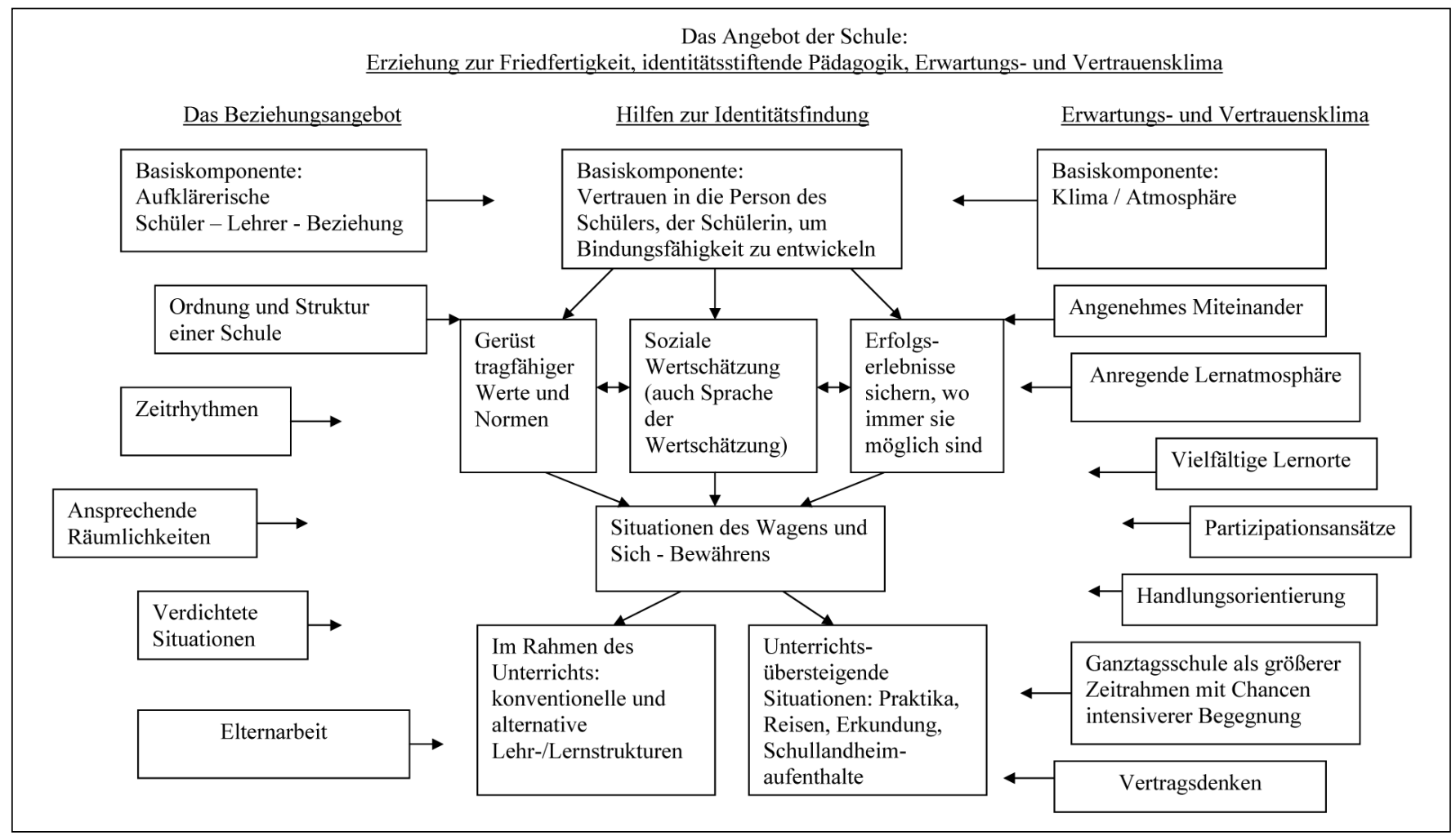


grund. Die Basiskomponente »Vertrauen « findet ihre tragenden Säulen in einem Gerüst tragfähiger Werte und Normen, in der Wertschätzung jeder Person. Und grundlegend ist: Ein Mensch muss mittelfristig Erfolgserlebnisse haben. Spannend ist dann, welche Situationen des Sich-Wagens und Sich-Bewährens eine Schule anbieten kann. Das Arrangement der Lernsituationen und immer wieder unterrichtsübersteigende Situationen gehören dazu. Die Künstlichkeit der Schule mit ihren immer wiederkehrenden Rezeptionsprozeduren wirkt eben häufig lebensfremd. Das Beziehungsangebot mit der Basiskomponente " aufklärerische Lehrer-Schüler-Beziehung « und den sich in der Übersicht anschließenden Gesichtspunkten flankieren den Mittelpunkt »Hilfen zur Identitätsfindung « ebenso wie das Erwartungsund Vertrauensklima einer Schule. Was die Zusammenarbeit von Lehrerinnen und Lehrern mit Schulsozialarbeiterinnen und Schulsozialarbeitern anbelangt, kann man auf dieser Folie immer prüfen, zu welchen Punkten nur gemeinsam gearbeitet werden kann und welche Komponenten arbeitsteilig verfolgt werden können.

Die zweite Ideen-Landkarte (Übersicht 2) strukturiert das Handlungsfeld Schule vom Kern der Sozialarbeit, hier Schulsozialarbeit, her. Sie soll deutlich machen, dass es jugendhilfespezifische Ansätze gibt, die das Leben und Lernen in der Schule wesentlich bereichern können, von Lehrerinnen und Lehrern nicht zu leisten sind und wesentlich an der Lebenswelt und Lebenslage von Kindern und Jugendlichen ansetzen.

Nicht der Gedanke nützlicher Ergänzung und Entlastung sollte dominieren das sei hier noch einmal wiederholt -, sondern dass das Eingehen auf Lebensweltgegebenheiten und konkrete Lebenslagen die Chance bietet, das schulische Lernen entweder überhaupt neu zu ermöglichen oder eben zu verbessern.

In der konkreten Ausmessung von Ansätzen und Aktivitäten werden sich dabei Varianzen von Schule zu Schule ergeben, weil die Problemlagen unterschiedlich sein werden. In einem Fall mögen vor allem Auffang- und Förderangebote notwendig sein. Im anderen Fall wird die Kinder- und Jugendarbeit schwerpunktmäßig Bereicherung und Anreicherung der vor allem kognitiv orientierten Schule sein. Im dritten Fall (vor allem in Hauptschulen) wird der Schwerpunkt "Jugendberufshilfe « der tragende sein. Im vierten Fall kann durch Vernetzung mit den örtlichen Angeboten der Jugendhilfe und der Jugendarbeit eine hilfreiche Strukturierung der Lebenswelt das Leben von Kindern und Jugendlichen aus Des- orientierung, Einsamkeit, Strukturlosigkeit herausführen. Auch mehraspektige Ansätze wird es geben.

\section{Kurze Bilanz}

Wenn das institutionell-organisatorische Denken vorherrscht - »Die Schule braucht jetzt aber dringend Sozialpädagogen; wir teilen denen ein paar zu «werden die angesprochenen Probleme bestehen bleiben. Die Wirksamkeit von Schulsozialarbeit wird von vornherein begrenzt sein. Das Nischendenken schafft sich zwar Handlungsfelder, ein integrativer Ansatz aber wird verfehlt.

Wenn man die Dringlichkeit der zu bearbeitenden Probleme gemeinsam identifiziert - schon das wäre ein Fortschritt -, wird man die Aufgabenfelder finden, die in einer Schule vordringlich zu bearbeiten sind. Der hier vertretene integrative Ansatz verlangt dann, die Verortung in den Ideen-Landkarten zu suchen, um konzeptionell bestimmen zu können, an welcher Stelle mit den in der Regel immer zu knappen Ressourcen sinnvoll angesetzt wird, um die schulische Arbeit zu verbessern.

Wenn die Nothelfer zu Schulmitarbeitern geworden sind, wird dies Indiz dafür sein, dass Schulsozialarbeit gelungen ist! Und die Schule wird dabei gewinnen.

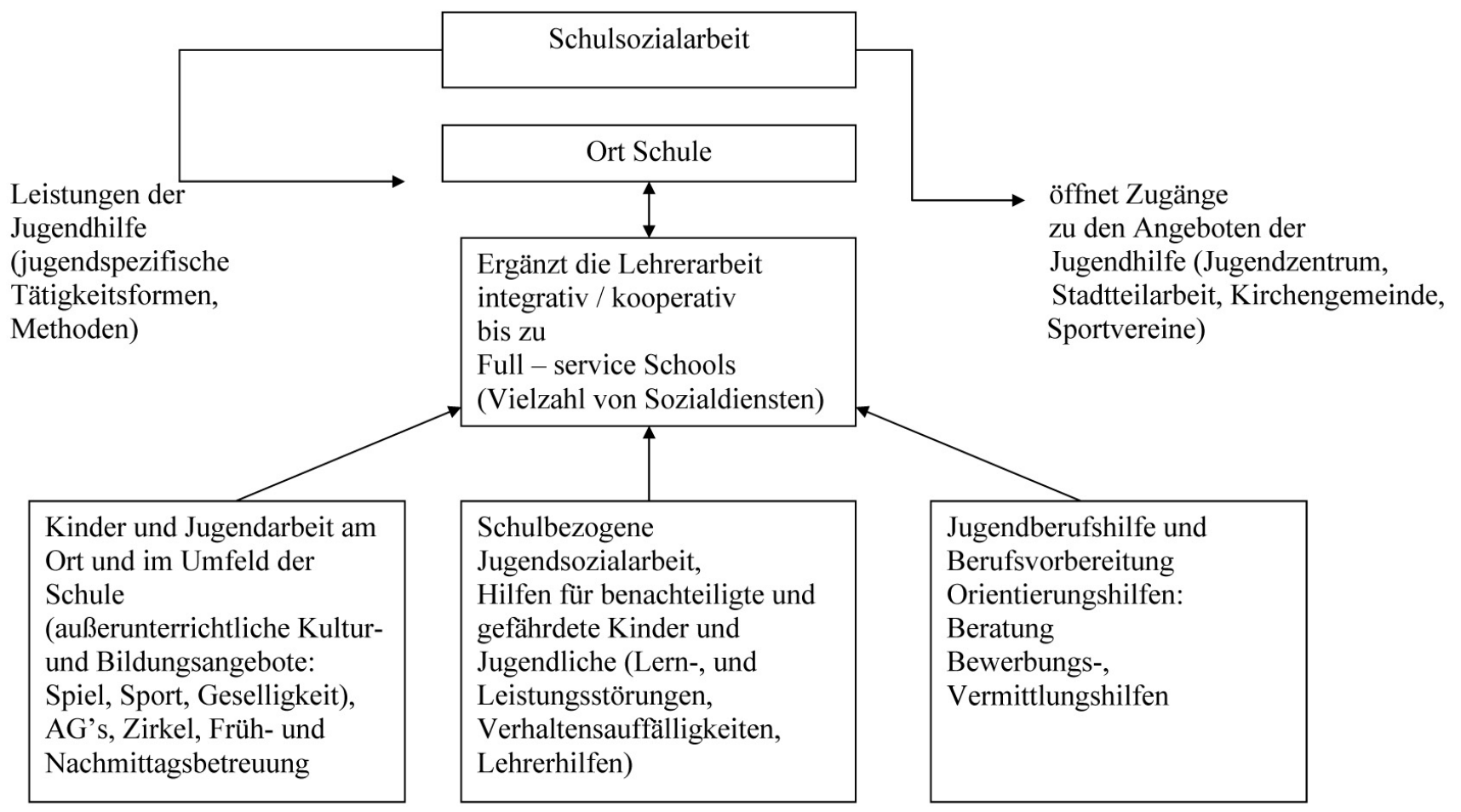




\section{Monitoring: Bewegungsmelder der Sozialen Arbeit}

In der Rubrik »Monitoring « nehmen Autorinnen und Autoren einzelne Arbeits- und Themenfelder der Sozialen Arbeit in den Blick um zu untersuchen, was sich dort Neues tut oder warum dort Praxis und Theorie auf der Stelle treten. Die Artikel sind eine aktuelle Ergänzung zu den wechselnden Schwerpunktthemen aus der Sozialen Arbeit in den Blättern der Wohlfahrtspflege. Eine Übersicht der bisherigen Beiträge in der Rubrik »Monitoring «:

Ein Masterplan für die kreative Stadt

Für eine Wiedergewinnung des Demokratischen

Von Konrad Hummel

Blätter der Wohlfahrtspflege 6/2007

\section{Die Macht des Lokalen}

Social Entrepreneurship, das Konzept der Verwirklichungs-

chancen und sozialer Mehrwert in der Sozialplanung

Von Veronika Hammer

Blätter der Woblfahrtspflege 6/2007

\section{Großer Aufholbedarf}

Zum Verhältnis sozialer Selbsthilfe und Sozialer Arbeit

Von Martin Stummbaum

Blätter der Wohlfahrtspflege 6/2007

Sorgenkind lokale Ökonomie

Ergebnisse der dritte Befragung in den Programmgebieten der

»Sozialen Stadt «

Von Christa Böhme und Thomas Franke

Blätter der Woblfahrtspflege 5/2007

Orientierungslos, mutlos, machtlos

Die Idee der Bürgergesellschaft hat es noch nicht zum gesellschaftlichen Leitbild geschafft - Das liegt auch an uns selbst

Von Stefan Nährlich

Blätter der Wohlfahrtspflege 4/2007

Synergielösungen für Sozialräume

Plädoyer für Fusionen kleiner Träger

Von Armin Wöhrle

Blätter der Woblfahrtspflege 4/2007

\section{Das Betreuungsgesetz droht zu scheitern}

Erforderlich sind eine konsequente Professionalisierung, mehr Kompetenzen für die Betreuungsbehörden, obligatorische Sozialgutachten sowie eine regelmäßige Berichterstattung

Von Wolf Crefeld

Blätter der Wohlfahrtspflege 3/2007

Wider die Individualisierung der Selbsthilfe

Karriere eines strapazierten Begriffs - Beispiel: Selbsthilfe

Wohnungsloser

Von Stefan Gillich

Blätter der Woblfahrtspflege 3/2007

Auf der Suche nach der guten Praxis

Die Qualitätsentwicklung in der Sozialen Arbeit - ein Überblick
Von Andrea Buckley und Karl-Heinz Boeßenecker Blätter der Woblfahrtspflege 2/2007

\section{Distanzierte Nähe}

Wie Wohlfahrtsverbände und traditionelle Milieus in Ostdeutschland (nicht) in Beziehung stehen

Von Peter-Georg Albrecht

Blätter der Wohlfahrtspflege 2/2007

Was Fachkräfte wissen müssen

Der Qualifikationsrahmen Soziale Arbeit

Von Peter Buttner und Ulrich Bartosch

Blätter der Woblfahrtspflege 1/2007, Seite 28-30

Das Format mit Inhalt füllen

Interview mit Wolf Rainer Wendt zum »Qualifikationsrahmen Soziale Arbeit «

Blätter der Wohlfahrtspflege 1/2007, Seite 29

Eltern stützen, Kinder schützen

Nach den Gesetzesänderungen:

Wie zukunftsfest ist das Kinder- und Jugendhilferecht?

Von Johannes Münder

Blätter der Wohlfahrtspflege 1/2007, Seite 31-32

Alles wird zur Dienstleistung

Die wirkungsorientierte Finanzierung von Jugendhilfe

Von Benjamin Landes

Blätter der Wohlfahrtspflege 1/2007, Seite 33-34

Schulsozialarbeit braucht mehr Rechte

Ein Berufsfeld der Sozialen Arbeit zwischen Lerntradition und Lebenswelt

Von Susanne Hartmann-Hanff

Blätter der Wohlfahrtspflege 1/2007, Seite 35-36

Qualitätssicherung zivilisiert den Wettbewerb

System und Arbeitsfelder im Vergleich

Von Roland Schmidt

Blätter der Wohlfahrtspflege 6/2006, Seite 230-232

Indikatoren gesucht

Neue Ansätze zur Integrationssteuerung in Deutschland

Von Kristin Schwarze

Blätter der Wohlfahrtspflege 6/2006, Seite 233-234

Die armen Kinder

Junge Menschen als die unsichtbaren Verlierer von Hartz IV

Von Gerda Holz

Blätter der Wohlfahrtspflege 5/2006, Seite 193-195

Der Jahresbezugspreis beträgt 58,- Euro (für Studierende und arbeitslose Bezieher auf jährlichen Nachweis 29,- Euro).

Die Einzelhefte können zum Preis von 11,- Euro zuzüglich Versandkosten und Mehrwertsteuer bezogen werden bei:

Nomos Verlagsgesellschaft, 76520 Baden-Baden, Telefon 07221 2104-39, Fax 07221210443,

E-Mailhohmann@nomos.de 